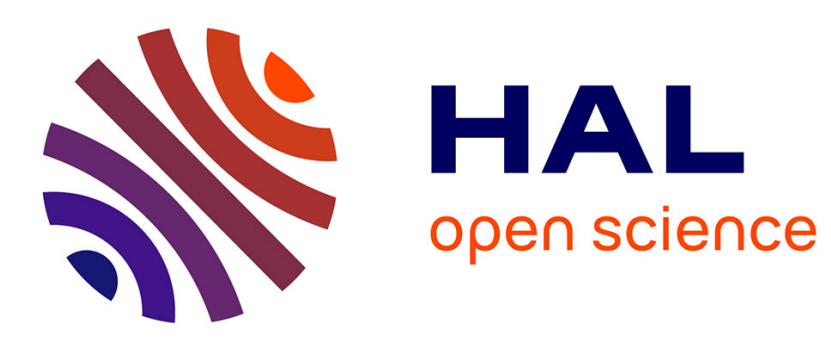

\title{
Representations with poles and cuts for the time-domain simulation of fractional systems and irrational transfer functions
}

Thomas Hélie, Denis Matignon

\section{- To cite this version:}

Thomas Hélie, Denis Matignon. Representations with poles and cuts for the time-domain simulation of fractional systems and irrational transfer functions. Signal Processing, 2006, vol. 86 ( $\left.\mathrm{n}^{\circ} 10\right)$, pp. 2516-2528. 10.1016/j.sigpro.2006.02.017 . hal-01373404

\section{HAL Id: hal-01373404 https://hal.science/hal-01373404}

Submitted on 28 Sep 2016

HAL is a multi-disciplinary open access archive for the deposit and dissemination of scientific research documents, whether they are published or not. The documents may come from teaching and research institutions in France or abroad, or from public or private research centers.
L'archive ouverte pluridisciplinaire HAL, est destinée au dépôt et à la diffusion de documents scientifiques de niveau recherche, publiés ou non, émanant des établissements d'enseignement et de recherche français ou étrangers, des laboratoires publics ou privés. 


\title{
Representations with poles and cuts for the time-domain simulation of fractional systems and irrational transfer functions
}

\author{
Thomas Hélie ${ }^{\mathrm{a}}$, Denis Matignon ${ }^{\mathrm{b}, *}$ \\ ${ }^{a}$ Laboratoire des Sciences et Technologie de la Musique et du Son, Equipe Analyse/Synthèse, CNRS UMR 9912 -Ircam, \\ Centre Georges Pompidou, 1, place Igor Stravinsky, 75004 Paris, France \\ ${ }^{\mathrm{b}}$ Laboratoire Traitement et Communication de l'Information, Département Traitement du Signal et des Images, \\ CNRS UMR 5141-Télécom Paris, 37-39, rue Dareau 75014 Paris, France
}

\begin{abstract}
Fractional differential systems are infinite-dimensional systems which are difficult to study and simulate: they can be represented with poles and cuts. This representation applies to a wider class of irrational transfer functions, and is most useful for signal processing purposes, such as frequency-domain and time-domain simulations: the approximations in low dimension which give the most striking numerical results are obtained through an optimization procedure, the parameters of which are meaningful from a signal point of view. Ten such systems of increasing complexity are thoroughly investigated.
\end{abstract}

Keywords: Infinite-dimensional systems; Irrational transfer functions; Time-domain simulation; Fractional differential systems; Diffusive representations

\section{Introduction}

There are many linear systems with irrational transfer functions, especially transfer functions of mathematical physics which involve fractional powers of the Laplace variable $s$. A wide class of such special functions can be found in e.g. the monograph by Duffy [1], some of which apply to infinite-dimensional representations [2]. A more specialized literature concentrates on fractional differential systems. We refer to recent works by

\footnotetext{
*Corresponding author.

E-mail addresses: Thomas.Helie@ircam.fr (T. Hélie), denis.matignon@enst.fr (D. Matignon).

$U R L$ : http://www.tsi.enst.fr/ matignon.
}

Carpinteri and Mainardi [3] and by Oustaloup [4] for physical models, and to the monograph by Miller and Ross [5] for the mathematical theory. Matignon and Montseny [6] provide examples that illustrate an interplay between signal processing, control theory and applications of such systems and their generalization.

Techniques for representing these systems in closed form, or in a manner more suitable for computation, not only in the frequency but also the time domain, need further study. The aim of this paper is to develop useful simulations from a point of view that lies half way between signal processing and control theory: briefly, complicated case studies will be introduced, analysed and simulated thoroughly. 


\section{Examples of systems involving fractional derivatives}

We select a family of 10 linear systems, which involve either fractional derivatives in the time domain, or fractional powers of the Laplace variable $s$ in their transfer function. Most of these cases stem from a physical example, which can be very simple or quite involved, but this is not the point in the present paper: here, we are more interested in presenting a hierarchy of fractional systems.

\subsection{An introductory example}

The following transfer function is irrational, but can be simply represented by a series of first-order systems:

$H_{1}(s)=\frac{\tanh (\sqrt{s})}{\sqrt{s}}=\sum_{n \in \mathbb{N}} \frac{2}{s+(n+1 / 2)^{2} \pi^{2}}$.

Such a transfer function appears in a boundary controlled and observed diffusion process in a bounded domain, see e.g. [2, Example 4.3.11]. Other examples of the same kind, which involve hyperbolic trigonometric functions and $\sqrt{s}$, for which a series expression is available, can be found in [1].

\subsection{Fractional integrals and derivatives}

The classical integral or derivative operators of fractional order also have irrational transfer functions, which cannot be represented by a series of first-order systems, but can be exactly represented by a continuous superposition of first-order systems (sometimes called diffusive representation) with some weight $\mu$, which can be computed analytically:

$H_{2}(s)=\frac{1}{s^{\beta}}, \quad 0<\mathfrak{R} e(\beta)<1$,

$H_{3}(s)=s^{\alpha}, \quad 0<\mathfrak{R} e(\alpha)<1$.

In the sequel, the output of system $H_{3}(s)=$ $s\left(1 / s^{1-\alpha}\right)$ will be considered as the (integer) timederivative of the output of system $\mathrm{H}_{2}$ with parameter $\beta=1-\alpha$; this simple remark will apply both for equivalent representations and simulation purposes. A technical well-posedness condition on this weight $\mu$ will distinguish between the two cases $\mathrm{H}_{2}$ and $H_{3}$. This condition of theoretical nature will also have numerical implications.

\subsection{Fractional differential systems}

A more complex combination of fractional derivatives gives rise to the so-called fractional differential systems, the transfer function of which reads either

$$
\begin{aligned}
H_{4}(s) & =R\left(s^{\alpha}\right)=\frac{Q\left(s^{\alpha}\right)}{P\left(s^{\alpha}\right)} \\
& =\frac{\sum_{l=0}^{l=q} b_{l} s^{l \alpha}}{\sum_{k=0}^{k=p} a_{k} s^{k \alpha}}, \quad 0<\Re e(\alpha)<1,
\end{aligned}
$$

or

$$
\begin{aligned}
H_{5}(s)= & \frac{\sum_{l=0}^{l=q} b_{l} s^{\beta_{l}}}{\sum_{k=0}^{k=p} a_{k} s^{\alpha_{k}}} \\
& \begin{array}{l}
0<\mathfrak{R} e\left(\beta_{l}\right)<\mathfrak{R} e\left(\beta_{l+1}\right), \\
0<\mathfrak{R} e\left(\alpha_{k}\right)<\mathfrak{R} e\left(\alpha_{k+1}\right) .
\end{array}
\end{aligned}
$$

The first case $H_{4}$ is known as fractional differential systems of commensurate order $\alpha$, which allows the use of some algebraic tools for equivalent representation, stability analysis and also simulation purposes.

On the contrary, the more general case $H_{5}$ is known as fractional differential systems of uncommensurate orders: for these systems, no algebraic tools can be applied, and both their analysis and simulation are quite involved.

Many results are known for these systems, as will be recalled later in Section 3.2.3.

\subsection{Diffusive systems}

Let us now consider examples which are neither a series of first-order systems, nor fractional differential systems, such as

$$
\begin{aligned}
& H_{6}(s)=\frac{\mathrm{e}^{-\sqrt{s}}}{\sqrt{s}}, \\
& H_{7}(s)=\mathrm{e}^{-\sqrt{s}} .
\end{aligned}
$$

Both can be decomposed on a continuous family of first-order systems with negative real poles $-\xi$, with a specific weight $\mu(\xi)$ playing exactly the same role as residues at the poles $s=-\xi$. The technical wellposedness condition on the weight $\mu$ will distinguish between the two cases $H_{6}$ and $H_{7}$; exactly for the same reason, a distinction was made between $\mathrm{H}_{2}$ and $H_{3}$ earlier in Section 2.2. 


\subsection{More complex systems}

Let us now consider some strange systems, the transfer function of which have poles of finite order and branching points with cuts to be chosen between them:

$H_{8}(s)=\frac{1}{\sqrt{s^{2}+1}}$

is the transfer function of the causal Bessel function of order zero $J_{0}(t)$, and it has been studied first in [7, Section 3.3], then in [8], and finally in [9, Example 3.1] with a new integral representation, which shows much freedom in the choice of the cuts between the two fixed branching points, namely $s= \pm i$.

Now, some more intricate transfer functions can easily be met on more complex examples,

$$
\begin{aligned}
H_{9}(s) & =\mathrm{e}^{s-\Gamma(s)} \text { with } \\
\Gamma(s) & =\sqrt{s^{2}+\varepsilon s^{\frac{3}{2}}+1} \text { and } \varepsilon>0, \\
H_{10}(s) & =\frac{2 \Gamma(s)}{s+\Gamma(s)} \mathrm{e}^{s-\Gamma(s)} .
\end{aligned}
$$

They are involved in the description of a 1-D wave equation in a flared duct of finite length, with viscothermal losses at the boundary: see e.g. [10] for a theoretical study of this model, [11,12] for simulation of these transfer functions, and [13, Chapter 9] for a study of the modal decomposition related to this model.

\section{Integral representations with poles and cuts}

We now investigate the general integral representations in the complex plane with poles and cuts: we present the general framework, and then apply it to the 10 examples presented in Section 2.

\subsection{General framework}

Many transfer functions can be decomposed as follows, in some right-half complex plane $\mathfrak{R} e(s)>a$,

$$
H(s)=\sum_{k=1}^{K} \sum_{l=1}^{L_{k}} \frac{r_{k, l}}{\left(s-s_{k}\right)}+\int_{\mathscr{C}} \frac{M(\mathrm{~d} \gamma)}{s-\gamma},
$$

which translates in the time domain into the following decomposition of the impulse response:

$$
\begin{aligned}
h(t)= & \sum_{k=1}^{K} \sum_{l=1}^{L_{k}} r_{k, l} \frac{1}{l !} t^{l-1} \mathrm{e}^{s_{k} t} \\
& +\int_{\mathscr{C}} \mathrm{e}^{\gamma t} M(\mathrm{~d} \gamma) \text { for } t>0 .
\end{aligned}
$$

The time-domain simulation of the finite-dimensional part of size $\sum_{k=1}^{K} L_{k}$ is really standard and will not be detailed in the sequel. The time-domain simulation of the infinite-dimensional part of these systems can quite easily be done through the following continuous family of first-order differential systems, parametrized by $\gamma \in \mathscr{C}$ :

$\partial_{t} \phi(\gamma, t)=\gamma \phi(\gamma, t)+u(t), \quad \phi(\gamma, 0)=0, \quad \forall \gamma \in \mathscr{C}$,

$y(t)=\int_{\mathscr{C}} \phi(\gamma, t) M(\mathrm{~d} \gamma)$,

which is nothing but an input $u$-state $\phi$-output $y$ representation of our system.

In all the integral equations above, $\mathscr{C}$ is a contour in some left-half complex plane, and $M$ is a measure on this contour. Once a parametrization has been chosen for the contour, the measure can be decomposed into different parts, such as a purely discrete part (Dirac measures at some points in some left-half complex plane) and an absolutely continuous part $\mu(\gamma)$ with respect to the Lebesgue measure $\mathrm{d} \gamma$. A straightforward interpretation can therefore be proposed: $\mu(\gamma)$ plays the role of the residue at the pole $s=\gamma$.

But of course, these representations make sense only if a so-called well-posedness condition is fulfilled, namely

$\int_{\mathscr{C}}\left|\frac{M(\mathrm{~d} \gamma)}{a+1-\gamma}\right|<\infty$.

We refer to [14, Sections 5 and 6] for the general theory and [15] for the implications of the wellposedness condition.

When $M$ has a density, an analytical computation of $\mu$ can be performed from $H$ across the cut, taking non-tangential limits; when $\mathscr{C}=\mathbb{R}^{-}$, we find with e.g. [7]:

$\mu(\xi)=\lim _{\varepsilon \rightarrow 0^{+}} \frac{1}{2 \mathrm{i} \pi}\{H(-\xi-\mathrm{i} \varepsilon)-H(-\xi+\mathrm{i} \varepsilon)\}$,

a formula which will be most useful in the sequel, namely in Section 3.2. This formula is obtained by inverting the Laplace transform of $H(s)$ on the Bromwich path defined in Fig. 1: the contribution of the part encircling $\mathbb{R}^{-}$leads to the gap measurement (16), whereas that of the circle pieces goes to zero as the radius $R$ tends to infinity, thanks to Jordan's lemma.

As already mentioned in Section 2, in some cases, since the well-posedness condition (15) is not met, 


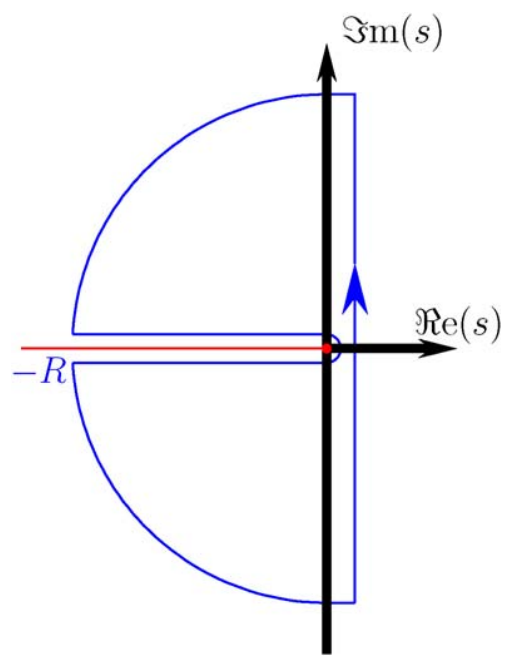

Fig. 1. Bromwich path for $H(s)$ with a cut on $\mathscr{C}=\mathbb{R}^{-}$.

an extension can be proposed, which is still meaningful in some larger mathematical framework, namely

$H(s)=s \int_{\mathscr{C}} \frac{\breve{M}(\mathrm{~d} \gamma)}{s-\gamma}+H(0)$,

where $\breve{M}$ is the measure associated to $\breve{H}(s)=$ $(H(s)-H(0)) / s$, and which gives rise to the following input $u$-state $\phi$-output $z$ representation in the time domain:

$\partial_{t} \phi(\gamma, t)=\gamma \phi(\gamma, t)+u(t), \quad \phi(\gamma, 0)=0, \quad \forall \gamma \in \mathscr{C}$,

$z(t)=\int_{\mathscr{C}} \partial_{t} \phi(\gamma, t) \check{M}(\mathrm{~d} \gamma)+H(0) u(t)$.

Let us now go back to our examples and see how they fit in the general framework.

\subsection{Choice of the cuts and computation of the} weights for the examples

\subsubsection{An introductory example}

Choosing $\mathscr{C}=\mathbb{R}^{-}$leads to $M_{1}=\sum_{n \in \mathbb{N}} 2 \delta(\xi-$ $\left.\left(n+\frac{1}{2}\right)^{2} \pi^{2}\right)$ with $\gamma=-\xi$, and (15) is fulfilled.

\subsubsection{Fractional integrals and derivatives}

Choosing $\mathscr{C}=\mathbb{R}^{-}$and $\gamma=-\xi$ leads to $\mu_{2}(\xi)=(\sin (\beta \pi) / \pi)\left(1 / \xi^{\beta}\right)$, which fulfills $(15)$.

But $H_{3}$ must be realized with an extension: $H_{3}(0)=0$ and $\breve{\mu}_{3}=\mu_{2}$ with the particular choice $\beta=1-\alpha$; thus, $\breve{\mu}_{3}$ now fulfills (15).

\subsubsection{Fractional differential systems}

3.2.3.1. Commensurate orders. Following e.g. [16, Section 2.2], one can decompose the impulse response of system $\mathrm{H}_{4}$ into a finite sum of special functions, namely Mittag-Leffler functions (defined by $\mathscr{E}_{\alpha}(\lambda, t)=t_{+}^{\alpha-1} \sum_{k=0}^{\infty}\left(\left(\lambda t_{+}^{\alpha}\right)^{k} / \Gamma((k+1) \alpha)\right)$ when the roots of $P$ in (4) are simple, see [17]). Hence, the transfer function reads $H_{4}(s)=$ $\sum_{n=1}^{p} r_{n}\left(s^{\alpha}-\lambda_{n}\right)^{-1}$, and the impulse response reads

$h_{4}(t)=\sum_{n=1}^{p} r_{n} \mathscr{E}_{\alpha}\left(\lambda_{n}, t\right)$.

This decomposition looks finite dimensional, but the following remarks apply:

- from a numerical point of view, these special functions are difficult to compute in the whole complex plane (since $\lambda_{n} \in \mathbb{C}$ ); even in the case $\alpha=\frac{1}{2}$, where the function is easily related to the classical error function, the argument is not limited to $\mathbb{R}$, and this makes the problem difficult;

- this decomposition allows an algebraic knowledge of the poles and residues (namely $s_{n}=\left(\lambda_{n}\right)^{1 / \alpha}$ and $r_{n}=(1 / \alpha) \lambda_{n}^{(1 / \alpha)-1}$, but only for those $\lambda_{n}$ satisfying $\left.\left|\arg \left(\lambda_{n}\right)\right|<\alpha \pi\right)$;

- any such Mittag-Leffler function has a representation with a pole and a cut on $\mathscr{C}=\mathbb{R}^{-}$, with a weight which can be computed exactly when $s_{n} \in \mathbb{C} \backslash \mathbb{R}^{-}$:

$$
\mu_{\alpha, \lambda_{n}}(\xi)=\frac{\sin (\alpha \pi)}{\pi} \frac{\xi^{\alpha}}{\xi^{2 \alpha}-2 \lambda_{n} \cos (\alpha \pi) \xi^{\alpha}+\lambda_{n}^{2}} .
$$

This is the reason why, at least for simulation purposes, the distinction between commensurate and uncommensurate orders proves a bit artificial.

3.2.3.2. Uncommensurate orders. Now, following [7, Section 2.3, 18], for both cases, the following decomposition can be written down for system $\mathrm{H}_{5}$ :

$$
\begin{aligned}
h_{5}(t)= & \sum_{k=1}^{K} \sum_{l=1}^{L_{k}} r_{k, l} \frac{1}{l !} t^{l-1} \mathrm{e}^{s_{k} t} \\
& +\int_{0}^{\infty} \mu_{5}(\xi) \mathrm{e}^{-\xi t} \text { for } t>0 .
\end{aligned}
$$

The following remarks apply to the previous decomposition:

- there is only a finite number of poles, as proved in $[19,20]$; 
- we have an analytical knowledge of $\mu_{5}$, namely (see $[7,16])$ :

$\mu_{5}(\xi)=\frac{1}{\pi} \frac{\sum_{k=0}^{p} \sum_{l=0}^{q} a_{k} b_{l} \sin \left(\left(\alpha_{k}-\beta_{l}\right) \pi\right) \xi^{\alpha_{k}+\beta_{l}}}{\sum_{k=0}^{p} a_{k}^{2} \xi^{2 \alpha_{k}}+\sum_{0 \leqslant k<l \leqslant p} 2 a_{k} a_{l} \cos \left(\left(\alpha_{k}-\alpha_{l}\right) \pi\right) \xi^{\alpha_{k}+\alpha_{l}}} ;$

- still, the case of poles on the cut $\mathbb{R}^{-}$is difficult, but it can be put in a somewhat larger framework, involving measures or distributions: in this case, the integral term in (22) is to be understood in a generalized sense (see e.g. [13, pp 71-73]). Yet, there is another strategy of representation, which consists in moving the cut between the same branching points $(s=0$ and $s=-\infty)$, so as to avoid the singularities, see e.g. [8].

\subsubsection{Diffusive systems}

Choosing $\mathscr{C}=\mathbb{R}^{-}$and $\gamma=-\xi$ leads to $\mu_{6}(\xi)=$ $\cos (\sqrt{\xi}) / \pi \sqrt{\xi}$, which fulfills (15).

But $\mathrm{H}_{7}$ must be realized with an extension: $H_{7}(0)=1$ and $\breve{\mu}_{7}=\sin (\sqrt{\xi}) / \pi \xi$; thus, $\breve{\mu}_{7}$ now fulfills (15).

\subsubsection{More complex systems}

For $\mathrm{H}_{8}$, we now have two finite branching points, thus many cuts can be proposed, we will only consider the two lines parallel to $\mathbb{R}^{-}$stemming from $\pm \mathrm{i}: \gamma^{ \pm}(\xi)= \pm \mathrm{i}-\xi$. Following [7, Section 3.3], we get

$\mu_{8}^{ \pm}(\xi)=\frac{1}{\pi \sqrt{\xi}} \frac{1}{\sqrt{ \pm 2 \mathrm{i}-\xi}}$,

with $\sqrt{s}$ uniquely defined for $s \in \mathbb{C} \backslash \mathbb{R}^{-}$as the analytic continuation of $\sqrt{x}$ for $x \in \mathbb{R}^{+}$. Once again, (15) is fulfilled.

For $H_{9}$ and $H_{10}$, also with three finite branching points $\left(0, s_{1}\right.$ and $\overline{s_{1}}$ with $\left.\mathfrak{R} e\left(s_{1}\right)<0\right)$, two different cuts will be investigated: either three horizontal cuts parallel to $\mathbb{R}^{-}$namely $\mathscr{C}=\mathbb{R}^{-} \cup\left(s_{1}+\mathbb{R}^{-}\right) \cup\left(\overline{s_{1}}+\right.$ $\left.\mathbb{R}^{-}\right)$as displayed in Fig. 2 (1)a and (1) b, or a cross-cut made of the segment between the two branching points and the cut on $\mathbb{R}^{-}$, namely $\mathscr{C}=\mathbb{R}^{-} \cup\left[s_{1}, \overline{s_{1}}\right]$ as displayed in Fig. 2 (1)C and (1)d. More details can be found in [12].

\section{Finite-dimensional approximation and simulation of poles and cut-representation models}

In this section, we propose to approximate stable realizations of fractional systems and irrational transfer functions (1-10) with finite order differen- tial systems, by picking up a finite subset of points which belong to the cut $\mathscr{C}$ and the set of poles $\mathscr{P}$ of the original system. Here, two methods are investigated to get such finite-order approximations: the first one relies on a convergent interpolation of the state $\phi$; the second one is based on an optimization procedure of a specially designed criterion. Corresponding numerical results are compared in both the frequency and the time domains.

Note that these finite-order approximations can also be described as pole-zero filters (see [4]), for which only pole placement is chosen. In our case, the location of the zeros is a consequence of either the interpolation or the optimization procedures. This holds true also for the discrete-time versions presented in Section 4.5.

\subsection{Approximation by interpolation of the state $\phi$}

A first method consists in approximating $\phi(\gamma, t)$, $\gamma \in \mathscr{C}$ by

$\widetilde{\phi}(\gamma, t)=\sum_{m=1}^{M} \phi(\gamma, t) \Lambda_{m}(\gamma)$,

where $\left\{\Lambda_{m}\right\}_{1 \leqslant m \leqslant M}$ defines continuous piecewise linear interpolating functions which are non-zero on the piece $] \gamma_{m-1}, \gamma_{m+1} L_{\mathscr{C}}$ of the cut $\mathscr{C}$ and such that $\Lambda_{m}\left(\gamma_{m}\right)=1 ;\left(\gamma_{m}\right)_{0 \leqslant m \leqslant M_{\mathscr{C}}}$ are sorted with respect to the oriented cut $\mathscr{C}$. Convergence results can be proven, e.g. see [13] for the purely diffusive case $\gamma=-\xi \in \mathbb{R}^{-}$.

Realization (13)-(14) yields the first-order linear system of dimension $M$

$\partial_{t} \widetilde{\phi}_{m}(t)=\gamma_{m} \widetilde{\phi}_{m}(t)+u(t), \quad 1 \leqslant m \leqslant M$,

$y(t)=\sum_{m=1}^{M} \widetilde{\mu}_{m} \widetilde{\phi}_{m}(t)$

with

$\widetilde{\mu}_{m}=\int_{\left[\gamma_{m-1}, \gamma_{m+1}\right]_{\mathscr{G}}} \mu(\gamma) \Lambda_{m}(\gamma) \mathrm{d} \gamma, \quad 1 \leqslant m \leqslant M$.

Contribution of poles $\gamma \in \mathscr{P}$ can be performed in the same way with standard finite-order systems which are not detailed here.

\subsection{Remark on approximations preserving the hermitian symmetry property}

For systems which map real inputs to real outputs, transfer functions satisfy the hermitian 


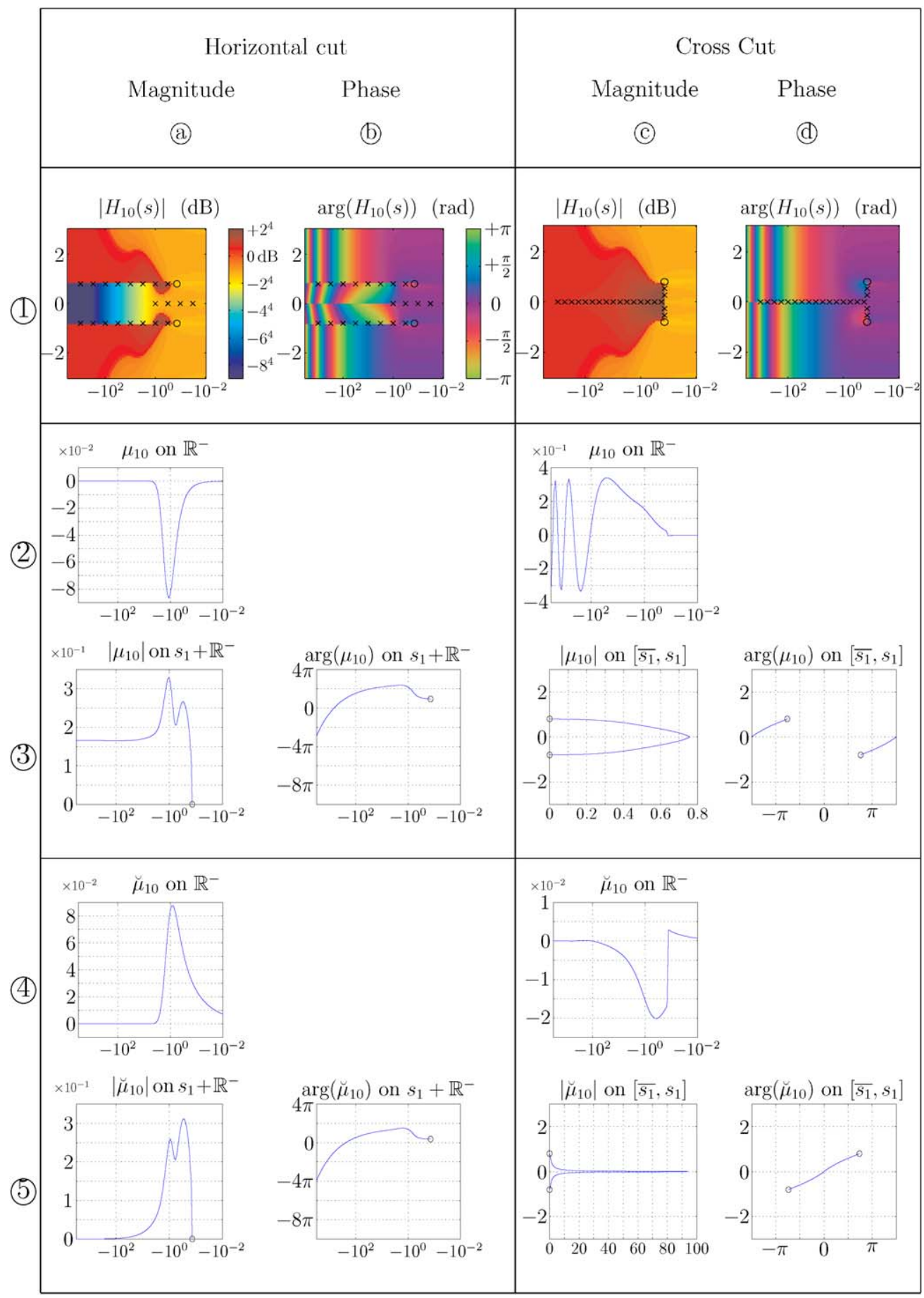

Fig. 2. Magnitude and phase of $H_{10}, s \in \mathbb{C}_{0}^{-}$are represented in (1) for horizontal cuts (a,b) and cross-cut (c,d). The corresponding (realvalued) $\mu_{10}$ computed on $\mathbb{R}^{-}$are in (2)a, c. The corresponding (complex-valued) are computed on $s_{1}+\mathbb{R}^{-}$(3)a: modulus, (3) b: phase) and on $\left[\overline{s_{1}}, s_{1}\right]$ (3): magnitude, (3)d: phase), respectively. The $X$-axis of (1), (2) and (3)a, b correspond to $-\xi=\mathfrak{R e}(s)<0$ on a log-scale, and the $Y$-axis of (1), (3) c, d to $\omega=\operatorname{Im}(s)$ on a linear scale. Circles $(o)$ represent branching points and crosses $(\times)$ the pole placement for further approximations. The weights $\mu$ in (2) c and (3)a do not decrease when $-\xi \rightarrow-\infty$, so that the well-posedness condition (15) is not satisfied. This problem disappears in (4), (5) for the extension by derivation $\breve{\mu}_{10}$, computed from $\breve{H}_{10}(s)$. After Hélie and Matignon [12]. 
symmetry $H(s)=\overline{H(\bar{s})}, s \in \mathbb{C}_{a}^{+}$. In this case, the same symmetry for the set of poles and the set $\mathscr{C}$ can be chosen for any analytic continuation of $(H(s)+\overline{H(\bar{s})}) / 2$. As a consequence, the set $\left(\gamma_{m}\right)_{1 \leqslant m \leqslant M}$ can be described by $-\xi=\left(-\xi_{j}\right)_{1 \leqslant j \leqslant J}$, $\xi_{j}>0$ for poles $\gamma$ lying on $\mathbb{R}^{-}$and by the complex conjugate pair $\underline{\gamma}=\left(\gamma_{k}\right)_{1 \leqslant k \leqslant K}$ and $(\bar{\gamma})$ with $\gamma_{k}=-\xi_{k}^{\prime}+\mathrm{i} \omega_{k}^{\prime}, \xi_{k}^{\prime}>0$, and $\omega_{k}^{\prime}>0$ otherwise. The approximation of (11) of dimension $M=J+2 K$ yields

$$
\begin{aligned}
\widetilde{H}_{\underline{\mu}}(s)= & \sum_{j=0}^{J} \frac{\mu_{j}}{s+\xi_{j}}+\sum_{k=0}^{K}\left[\mu_{k}^{R}\left(\frac{1}{s-\gamma_{k}}+\frac{1}{s-\overline{\gamma_{k}}}\right)\right. \\
& \left.+\mu_{k}^{I}\left(\frac{\mathrm{i}}{s-\gamma_{k}}+\frac{-\mathrm{i}}{s-\overline{\gamma_{k}}}\right)\right],
\end{aligned}
$$

where $\mu$ denotes the real vector $\left(\mu_{1}, \ldots, \mu_{J}\right.$, $\left.\mu_{1}^{R}, \ldots, \overline{\mu_{K}^{R}}, \mu_{1}^{I}, \ldots, \mu_{K}^{I}\right)^{t} \in \mathbb{R}^{J+2 K}, \mu_{j}$ and $\mu_{k}^{\prime}=\mu_{k}^{R}+$ $\mathrm{i} \mu \mu_{k}^{I}$ are the associated weights. The realness of $\underline{\mu}$ ensures hermitian symmetry.

\subsection{Approximation by optimization of a criterion}

The second method consists in a least-squares regularized optimization of the weights $\underline{\mu}$, by minimizing an appropriate distance between an exact transfer function $H(\mathrm{i} \omega)$ and its approximation $\widetilde{H}_{\mu}(\mathrm{i} \omega)$ in the frequency domain, see e.g. $[21,8]$.

$\mathscr{C}(\underline{\mu})=\int_{\mathbb{R}^{+}}\left|\widetilde{H}_{\underline{\mu}}(\mathrm{i} \omega)-H(\mathrm{i} \omega)\right|^{2} w(\omega) \mathrm{d} \omega$,

where the weight $w(\omega)$ can be uniform on the whole frequency range $(w(\omega)=1)$ for a classical $L^{2}$-norm, or frequency-dependent such as $w_{s}(\omega)=\left(1+\omega^{2}\right)^{s}$ for a Sobolev $H^{s}$-norm. In this latter case, the real parameter $s$ can be seen as a tuning parameter of the low and high frequency balance. But weights $w$ can also be viewed as the result of scalings: for instance, considering a logarithmic scale for frequencies, such as in Bode diagrams, results in choosing the measure $\mathrm{d} \ln \omega=\mathrm{d} \omega / \omega$, which amounts to use $w(\omega)=1 / \omega$.

In practice, such considerations can be used to build weights based on each application. For instance, $w(\omega)$ can be adapted and modified according to the following requirements:

- a bounded frequency range $\omega \in\left[\omega_{-}, \omega_{+}\right]$: $w(\omega) \mathbf{1}_{\left[\omega_{-}, \omega_{+}\right]}(\omega)$;

- a frequency log-scale: $w(\omega) / \omega$;

- a relative error measurement: $w(\omega) /|H(\mathrm{i} \omega)|^{2}$
- a relative error on a bounded dynamics: $w(\omega) /\left(\operatorname{Sat}_{H, \Theta}(\omega)\right)^{2}$ where the saturation function Sat $_{H, \Theta}$ with threshold $\Theta$ is defined by

$\operatorname{Sat}_{H, \Theta}(\omega)= \begin{cases}|H(\mathrm{i} \omega)| & \text { if }|H(\mathrm{i} \omega)| \geqslant \Theta_{H}, \\ \Theta_{H} & \text { otherwise, }\end{cases}$

with $\Theta_{H}=\Theta \sup _{\{\omega \mid w(\omega) \neq 0\}}|H(\mathrm{i} \omega)|$. Note that a $80 \mathrm{~dB}$-dynamics corresponds to $\Theta=10^{-4}$;

- a conversion for the extension as defined in (17): the distance between $H$ and $\widetilde{H}$ for $w(\omega)$ is the same as the distance between $\breve{H}$ and $\widetilde{H}$ for the adapted weight $\omega^{2} w(\omega)$.

All these requirements can be cumulated in the appropriate order.

Nevertheless, criterion (29) can lead to ill-conditioned problems. We use standard regularization techniques to cope with this [22], introducing the regularized criterion $\mathscr{C}_{\mathrm{R}}(\underline{\mu})$ :

$\mathscr{C}_{\mathrm{R}}(\underline{\mu})=\mathscr{C}(\underline{\mu})+\sum_{j=1}^{J} \varepsilon_{j}\left(\mu_{j}\right)^{2}+\sum_{k=1}^{K} \varepsilon_{k}^{\prime}\left(\left(\mu_{k}^{\mathrm{R}}\right)^{2}+\left(\mu_{k}^{I}\right)^{2}\right)$.

Numerically, criterion (29) is computed for angular frequencies increasing from $\omega_{1}=\omega_{-}$to $\omega_{N+1}=\omega_{+}$, using the approximation

$\mathscr{C}(\mu) \approx \sum_{n=1}^{N} w_{n}\left|\widetilde{H}_{\mu}\left(s_{n}\right)-H\left(s_{n}\right)\right|^{2}$,

with $w_{n}=\int_{\omega_{n}}^{\omega_{n+1}} w(\omega) \mathrm{d} \omega$ and $s_{n}=\mathrm{i} \omega_{n}$ for $1 \leqslant n \leqslant N$. Thus, with matrix notations, the criterion rewrites

$\mathscr{C}_{\mathrm{R}, \mathrm{L}}(\underline{\mu})=(\underline{M} \underline{\mu}-\underline{h})^{*} \underline{W}(\underline{M} \underline{\mu}-\underline{h})+\underline{\mu}^{t} \underline{E} \underline{\mu}$,

where $\underline{M}^{*}=\underline{M}^{t}$ denotes the transpose conjugate, and

- $\underline{M}$ is an $N \times(J+2 K)$ matrix, defined by $\bar{M}_{n, m}=\left[\mathrm{i} \omega_{n}+\xi_{m}\right]^{-1}$ for $\quad 1 \leqslant m \leqslant J$, by $M_{n, m}=\left[\mathrm{i} \omega_{n}-\gamma_{m-J}\right]^{-1}+\left[\mathrm{i} \omega_{n}-\overline{\gamma_{m-J}}\right]^{-1}$ for

$J+1 \leqslant m \leqslant J+K, \quad$ and by $M_{n, m}=\mathrm{i}\left[\mathrm{i} \omega_{n}-\gamma_{m-(J+K)}\right]^{-1}+\mathrm{i}\left[\mathrm{i} \omega_{n}-\bar{\gamma}_{m-(J+K)}\right]^{-1}$ for $J+K+1 \leqslant m \leqslant J+2 K$.

- $\underline{E}$ is a $(J+2 K) \times(J+2 K)$ real non-negative diagonal matrix, defined by $E_{m, m}=\varepsilon_{m}$ for $1 \leqslant m \leqslant J$ and by $E_{m, m}=E_{K+m, K+m}=\varepsilon_{m}^{\prime}$ for $J+1 \leqslant m \leqslant J+K$.

- $\underline{W}$ is the $N \times N$ diagonal matrix $\operatorname{diag}\left(w_{n}\right)$.

- $\underline{h}$ is the $N \times 1$ vector $h_{n}=H\left(s_{n}\right)$. 
Solving this least-squares problem with the constraint that $\underline{\mu}$ is real valued yields

$\underline{\mu}=\mathscr{M}^{-1} \mathscr{H}$,

where

$\mathscr{M}=\mathfrak{R e}\left(\underline{M}^{*} \underline{W} \underline{M}+\underline{E}\right)$ and $\mathscr{H}=\mathfrak{R e}\left(\underline{M}^{*} \underline{W} \underline{h}\right)$.

This result is obtained by decomposing the complex values as $x+\mathrm{i} y$, solving the problem and recomposing the result into the closed form (34).

\subsection{Results in the frequency domain}

Plots of $\mu(\gamma)$ and results for both approximations (27) and (34) are presented in Figs. 3-7 for $H_{1}$ to $H_{4}$, $H_{6}, H_{8}$ and in Fig. 2 for $H_{10}$. Two choices are illustrated for the optimization procedure: either the uniform weight, denoted by $W_{\text {unif }}$, or the weight resulting from a relative error measurement with a frequency logarithmic scale, denoted by $W_{\text {log,rel }}$. A general remark is that the approximation by optimization does not require the analytic computation of $\mu(\gamma)$ and, for a given pole placement $\left(\gamma_{m}\right)_{1 \leqslant m \leqslant M}$, it yields better results than those obtained by interpolation. Nevertheless, the analysis of $\mu(\gamma)$ is required to check the well-posedness condition (15) and it is useful to build the relevant pole placements $\left(\gamma_{m}\right)_{1 \leqslant m \leqslant M}$. This latter point is illustrated for $H_{6}$ in Fig. 6 .

\subsection{Time-domain simulations}

The finite-dimensional realizations of the approximated transfer functions are

$$
\begin{aligned}
& \partial_{t} \phi_{j}(t)=-\xi_{j} \phi_{j}(t)+u(t), \quad 1 \leqslant j \leqslant J, \\
& \partial_{t} \phi_{k}^{\prime}(t)=\left(-\xi_{k}^{\prime}+\mathrm{i} \omega_{k}^{\prime}\right) \phi_{k}^{\prime}(t)+u(t), \quad 1 \leqslant k \leqslant K,
\end{aligned}
$$

$$
\begin{aligned}
\widetilde{y}(t)= & \sum_{j=1}^{J} \mu_{j} \phi_{j}(t)+2 \mathfrak{R e} \sum_{k=1}^{K} \mu_{k}^{\prime} \phi_{k}^{\prime}(t), \\
\widetilde{z}(t)= & \sum_{j=1}^{J}\left(-\xi_{j} \breve{\mu}_{j}\right) \phi_{j}(t) \\
& +2 \Re \mathrm{e} \sum_{k=1}^{K}\left(-\xi_{k}^{\prime}+\mathrm{i} \omega_{k}^{\prime}\right) \breve{\mu}_{k}^{\prime} \phi_{k}(t) \\
& +\left[H(0)+\sum_{j=1}^{J} \breve{\mu}_{j}+2 \Re \mathrm{R} \sum_{k=1}^{K} \breve{\mu}_{k}^{\prime}\right] u(t),
\end{aligned}
$$
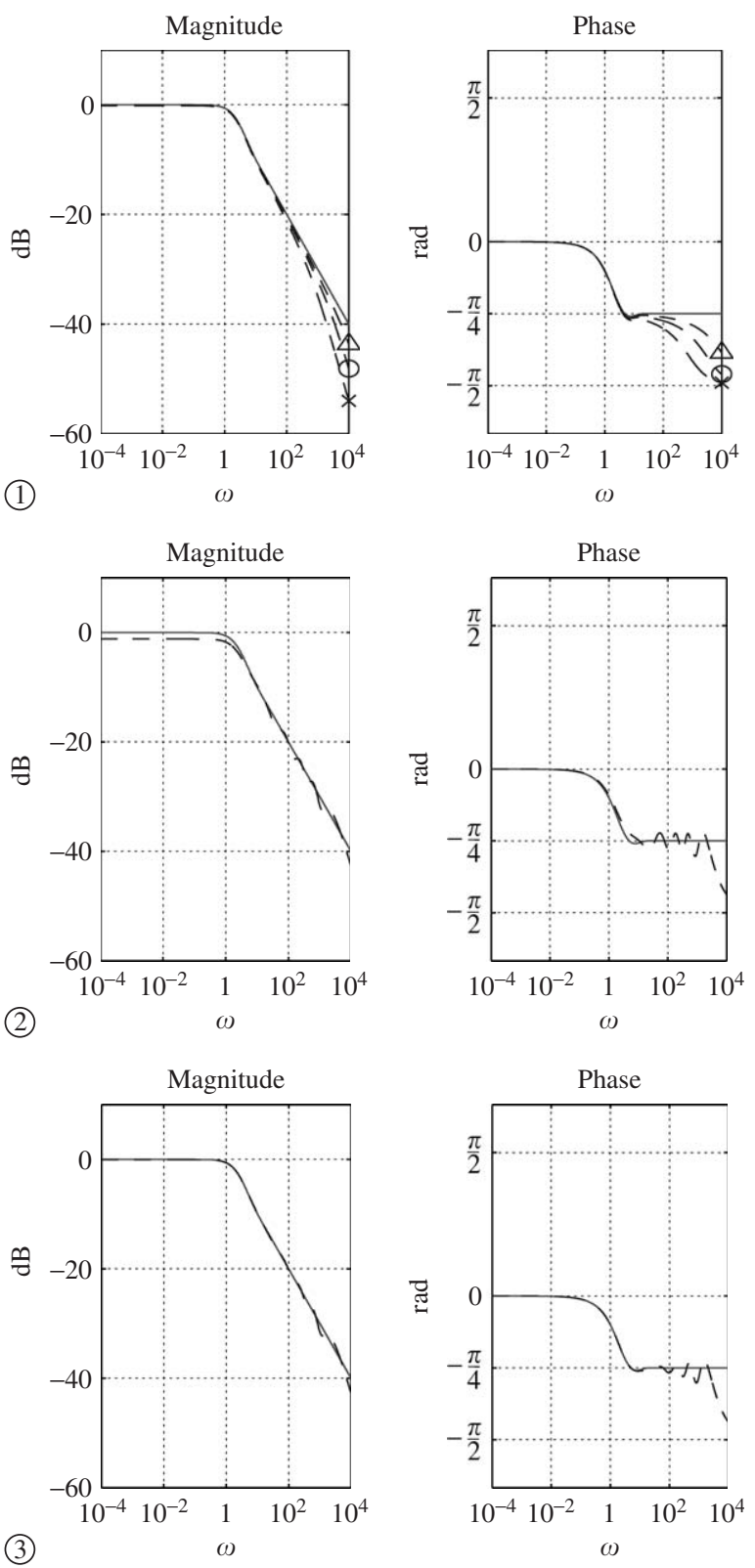

Fig. 3. Bode diagrams for transfer function $H_{1}$ : (1) exact (-), truncated series with the first $N$ poles, $N=10(\times), N=20(\circ)$ and $N=40(\triangle)$; (2) exact (-) and optimized (-) with $N=10$ and $W=W_{\text {unif }}$; (3) exact (-) and optimized (--) with $N=10$ and $W=W_{\text {log,rel }}$.

where $\widetilde{y}(t)$ stands for the output of a standard representation while $\widetilde{z}(t)$ stands for the output of an extension by derivation (17).

Approximating $u(t)$ by its sample and hold version, that is $u(t) \approx u\left(t_{n}\right)$ for $t_{n} \leqslant t<t_{n+1}$, Eqs. (35)-(38) 


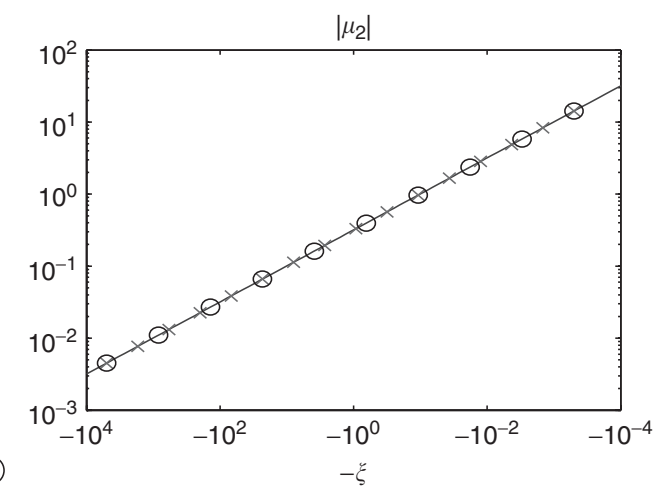

(1)
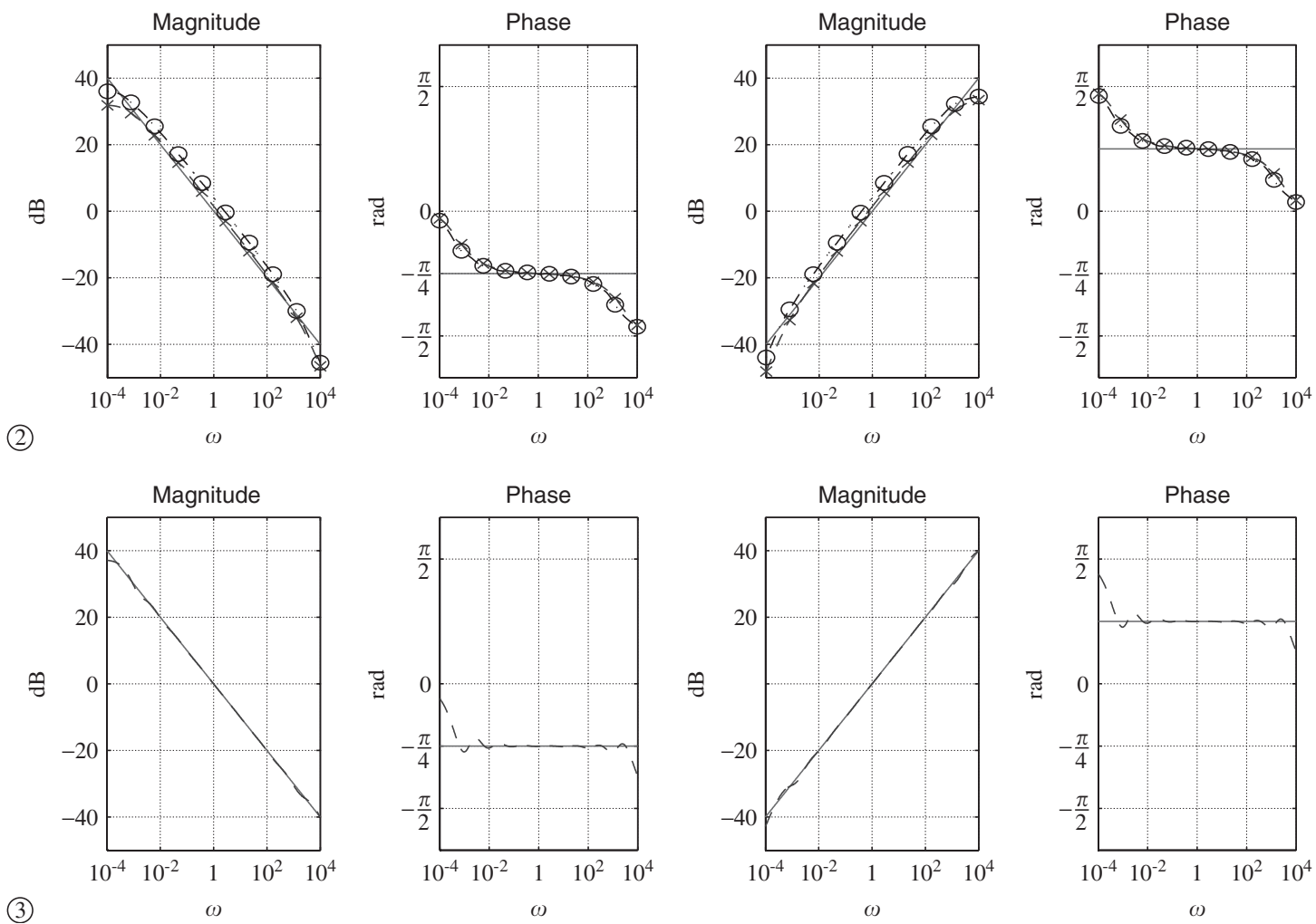

Fig. 4. Weight $\mu_{2}(\xi)=\breve{\mu}_{3}(\xi)$ for $\beta=1-\alpha=\frac{1}{2}(1, \mathrm{a})$ and two logarithmic pole placements with $N=10(o), N=16(\times)$ between $\xi_{\min }=$ $5 \times 10^{-4}$ and $\xi_{\max }=5 \times 10^{3}$. The corresponding Bode diagrams are in column (a) for $H_{2}$ and in (b) for $H_{3}$. Line (2) gives the exact Bode diagrams (-), and the result of interpolations $(o, \times)$. Line 3 gives the exact Bode diagrams (-), and the result of optimization (--) for the case $N=10$ with $W=W_{\text {log, rel }}$.

become, in the discrete-time domain,

$\phi_{j}\left(t_{n}\right)=\alpha_{j} \phi_{j}\left(t_{n-1}\right)+\frac{\alpha_{j}-1}{-\xi_{j}} u\left(t_{n-1}\right)$,

$\phi_{k}^{\prime}\left(t_{n}\right)=\alpha_{k}^{\prime} \phi_{k}^{\prime}\left(t_{n-1}\right)+\frac{\alpha_{k}^{\prime}-1}{-\xi_{k}^{\prime}+\mathrm{i} \omega_{k}^{\prime}} u\left(t_{n-1}\right)$,

$\widetilde{y}\left(t_{n}\right)=\sum_{j=1}^{J} \mu_{j} \phi_{j}\left(t_{n}\right)+2 \mathfrak{R} \sum_{k=1}^{K} \mu_{k}^{\prime} \phi_{k}^{\prime}\left(t_{n}\right)$,

$$
\begin{aligned}
\widetilde{z}\left(t_{n}\right)= & \sum_{j=1}^{J}\left(-\xi_{j} \breve{\mu}_{j}\right) \phi_{j}\left(t_{n}\right) \\
& +2 \mathfrak{R e} \sum_{k=1}^{K}\left(-\xi_{k}^{\prime}+\mathrm{i} \omega_{k}^{\prime}\right) \breve{\mu}_{k}^{\prime} \phi_{k}^{\prime}\left(t_{n}\right) \\
& +\left[H(0)+\sum_{j=1}^{J} \breve{\mu}_{j}+2 \mathfrak{R e} \sum_{k=1}^{K} \mu_{k}^{\prime}\right] u\left(t_{n}\right),
\end{aligned}
$$



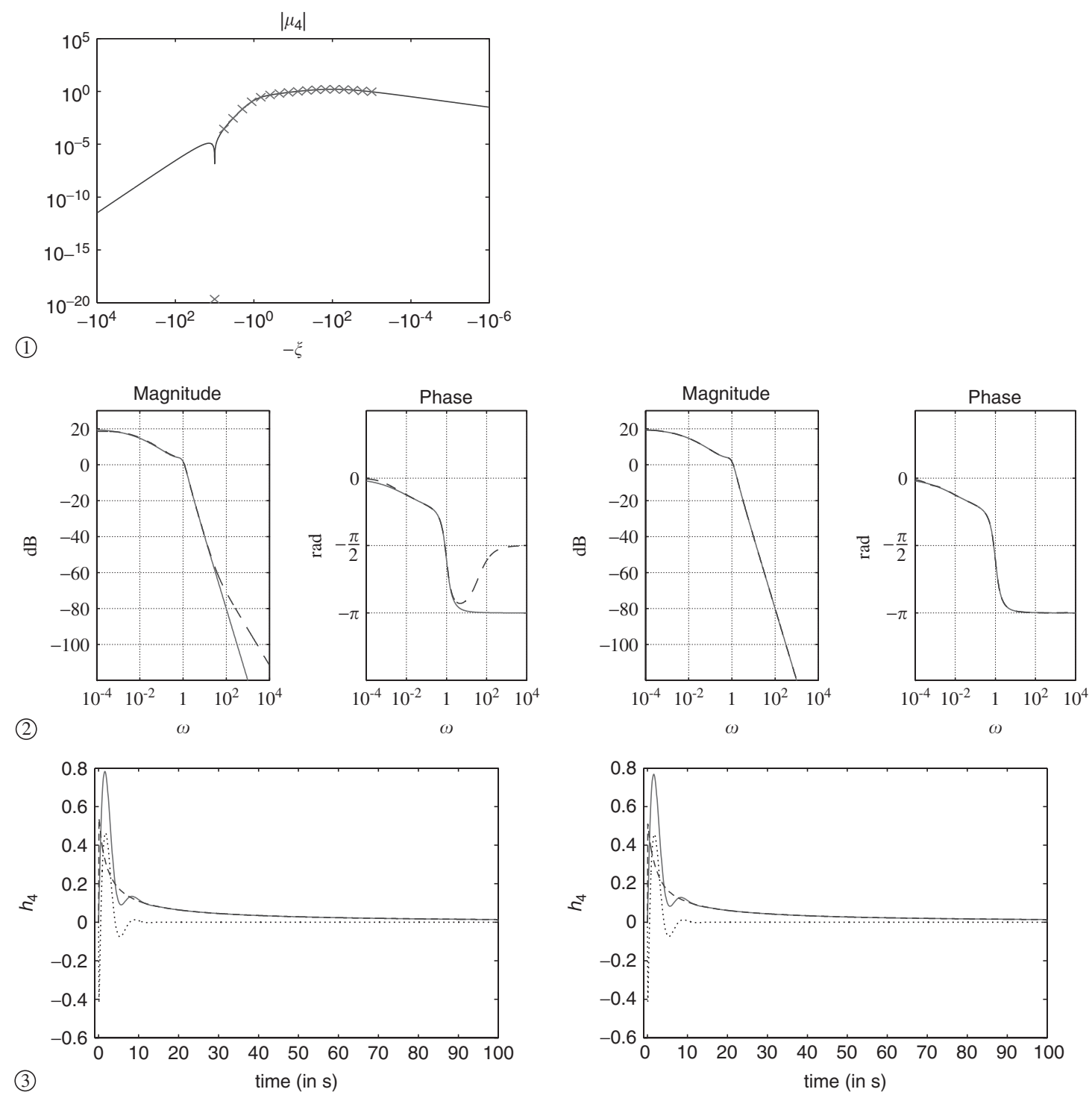

Fig. 5. Weight $\mu_{4}(\xi)$ with [13] $\alpha=\frac{1}{2}, Q(\sigma)=1$ and $P(\sigma)=\sigma^{4}+0.1 \sigma^{3}+\sigma+0.1(1$, a) and logarithmic pole placement $(\times)$ with $N=18$, between $\xi_{\min }=1 \times 10^{-3}$ and $\xi_{\max }=10$. Column (a) corresponds to exact (-) and interpolated (- - ) results, and column (b) to exact (-) and

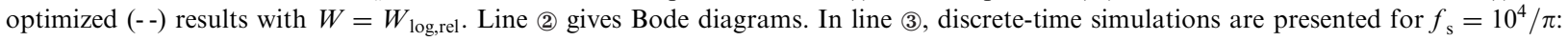
$h_{4}(t)$ are in solid lines (-), the diffusive part in dashed lines (- $)$ and the second order oscillatory part in dotted lines (:).

where $t_{n}=n T_{s}, T_{s}$ is the sampling period, $\alpha_{j}=\mathrm{e}^{-\xi_{j} T_{s}}$ and $\alpha_{k}^{\prime}=\mathrm{e}^{\left(-\xi_{k}^{\prime}+\mathrm{i} \omega_{k}^{\prime}\right) T_{s}}$.

The impulse responses $h_{4}\left(t_{n}\right), h_{6}\left(t_{n}\right), h_{8}\left(t_{n}\right)$ and $h_{10}\left(t_{n}\right)$ are simulated thanks to these recursive equations for both the approximations by interpolation and by optimization. The results for approximations by optimization are still better than those obtained by interpolation. They are presented in Figs. 5-7.

\section{Perspectives}

Some interesting questions remain still open. First, the choice of the cut between fixed branching points in the left-half complex plane can be made on different criteria, but it is not easy to know a priori which representation fits best. Second, once a cut has been chosen, what is the optimal pole placement on it, in order to reduce the infinite-dimensional 


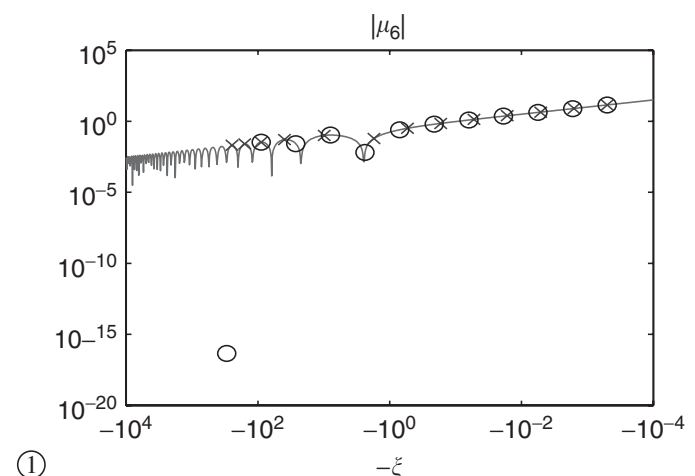

(1)

$-\xi$
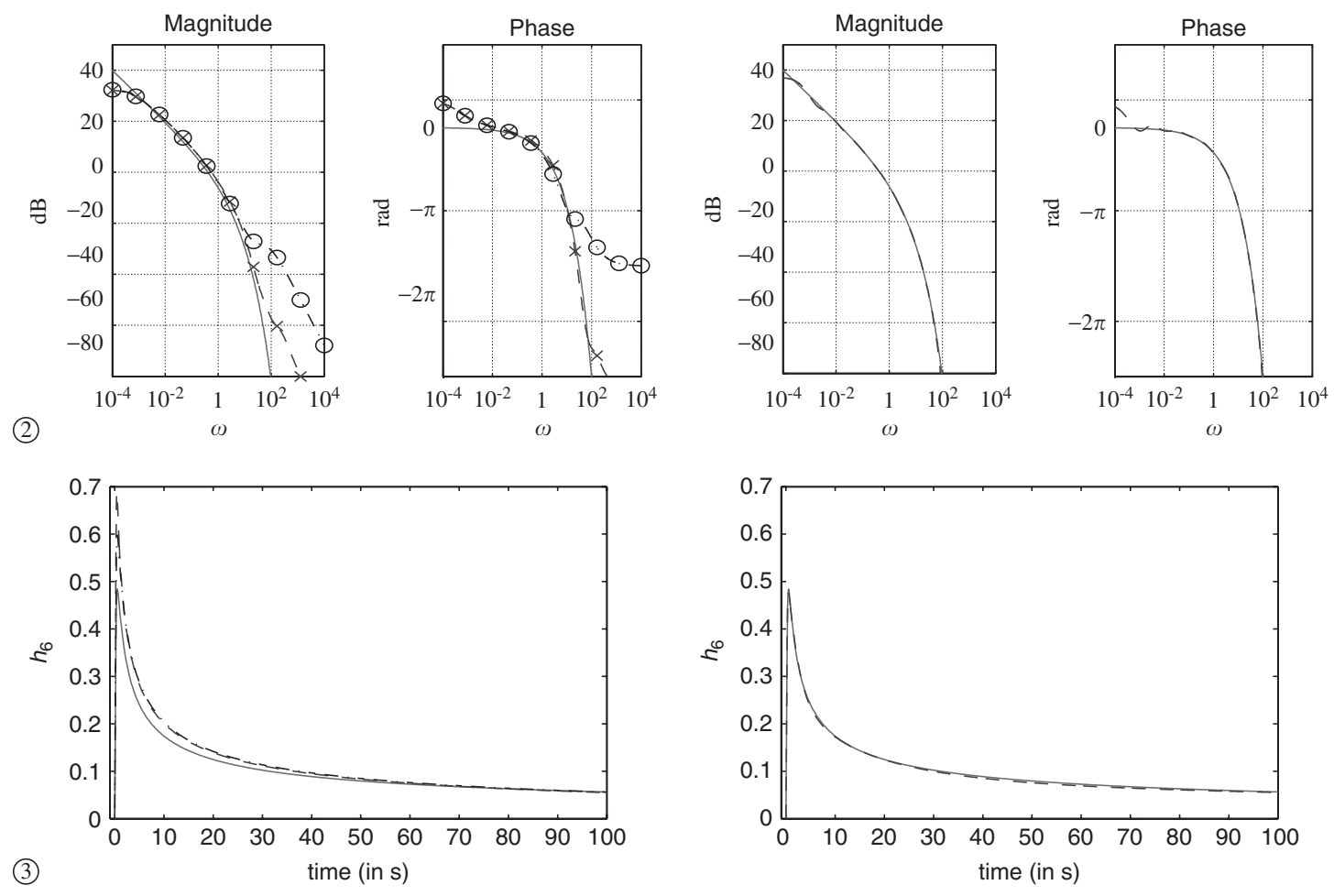

Fig. 6. Weight $\mu_{6}(\xi)$ and two pole placements with $N=12$ between $\xi_{\min }=5 \times 10^{-4}$ and $\xi_{\max }=3 \times 10^{2}$ : one is purely logarithmic (o), whereas the second $(\times)$ is modified to match with some maxima of $\left|\mu_{6}\right|$. Column (a) corresponds to exact $(-)$ and interpolated $(o, \times)$ results, and column (b) to exact (-) and optimized (- ) results with $W=W_{\text {log,rel }}$ and the pole placement ( $\times$ ). Line (2) gives Bode diagrams. In line (3), discrete-time simulations are presented for $f_{\mathrm{s}}=10^{4} / \pi$ : the exact impulse response $h_{6}(t)$ is in solid lines (-); the impulse responses computed for the interpolations $(o, x)$ are very similar, and both represented with the dashed lines in (3)a; in (3) b, the exact and optimized impulse responses superimpose.

system to a finite-order approximation (more suitable for time-domain simulation)? Both these questions are quite involved from a theoretical point of view; nevertheless, they must be taken into consideration, for they can have serious numerical consequences for the finite-dimensional approximation of our fractional systems.
Finally, it is of interest to enlarge the class of irrational transfer functions by allowing for delay systems to be present: so far, they have not been taken into account in our framework; even if some theoretical results are available, this will be a wide open direction of research concerning representation and simulation of such systems. 

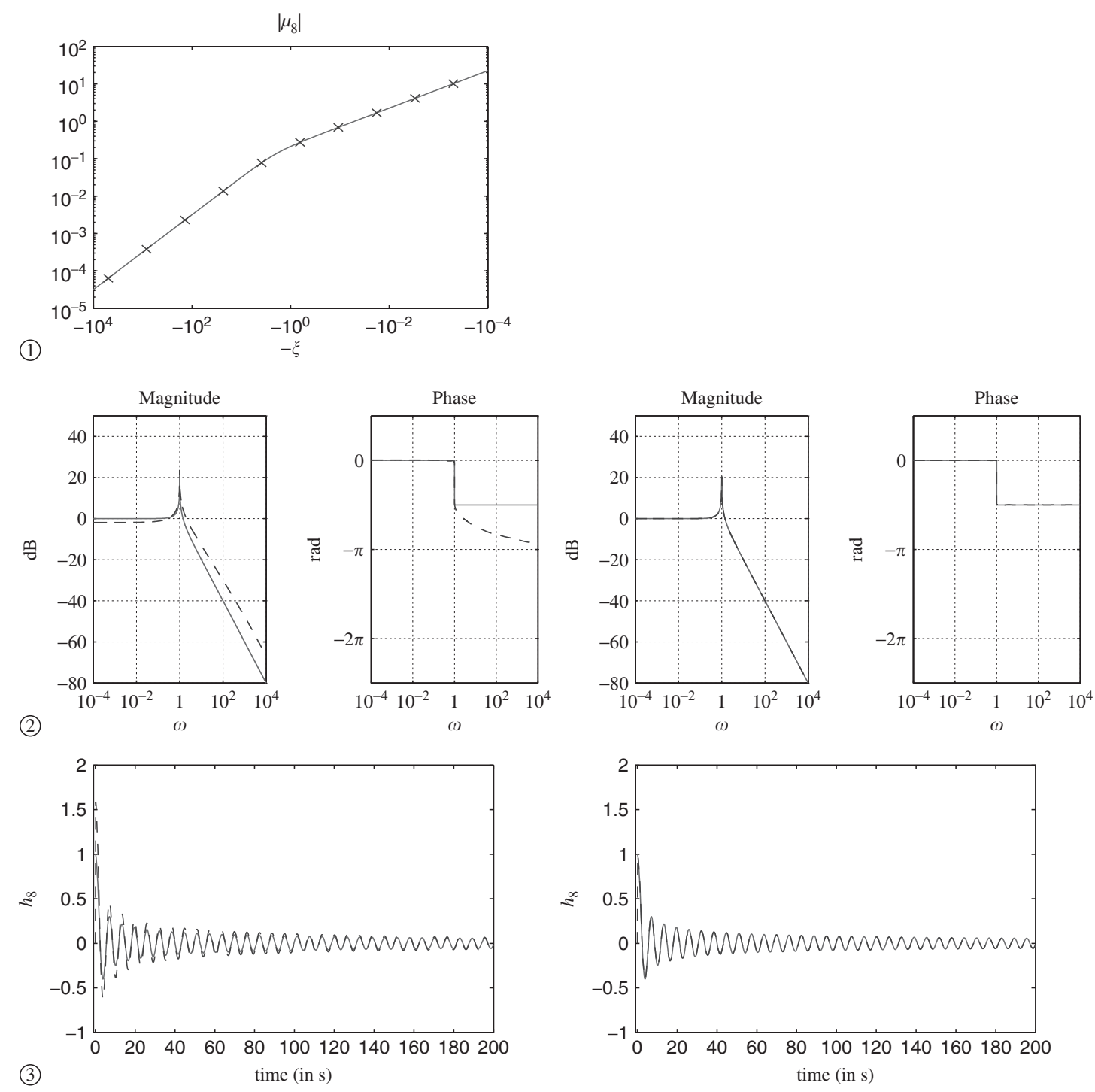

Fig. 7. Weight $\mu_{8}(\xi)$ for a logarithmic pole placement $(\times)$ with $N=10$ between $\xi_{\min }=5 \times 10^{-4}$ and $\xi_{\max }=5 \times 10^{3}$. Column (a) corresponds to exact (-) and interpolated (--) results, and column (b) to exact (-) and optimized (- ) results with $W=W_{\text {log,rel }}$. Line (2) gives Bode diagrams. In line (3), discrete-time simulations are presented for $f_{\mathrm{s}}=10^{4} / \pi$ : the exact $h_{8}(t)$ is in solid lines (-), the approximated impulse responses are in dashed lines (--).

\section{Conclusion}

A powerful and flexible method of simulation for fractional systems has been presented: it uses a simple optimization procedure with parameters that are meaningful from a signal processing point of view, and it enables a low cost simulation, both in the frequency domain and in the time domain. From a theoretical point of view, this method is based on a representation with poles and cuts, which generalizes the so-called diffusive representations. A family of 10 such systems, among which fractional differential systems, is presented throughout the paper, which clearly illustrates the generality, the flexibility and the power of this method.

\section{References}

[1] D.G. Duffy, Transform Methods for Solving Partial Differential Equations, CRC Press, Boca Raton, FL, 1994. 
[2] R.F. Curtain, H.J. Zwart, An introduction to infinitedimensional linear systems theory, Texts in Applied Mathematics, vol. 21, Springer, Berlin, 1995.

[3] A. Carpinteri, F. Mainardi (Eds.), Fractals and Fractional Calculus in Continuum Mechanics, CISM Courses and Lectures, vol. 378, Springer, Berlin, 1997.

[4] A. Oustaloup, Systèmes asservis linéaires d'ordre fractionnaire, Série Automatique, Masson, 1983.

[5] K.S. Miller, B. Ross, An Introduction to the Fractional Calculus and Fractional Differential Equations, Wiley, New York, 1993.

[6] D. Matignon, G. Montseny (Eds.), Fractional Differential Systems: Models, Methods and Applications, ESAIM: Proceedings, vol. 5, SMAI, URL: http://www.edpsciences. org/articlesproc/Vol.5/, 1998.

[7] D. Matignon, Stability properties for generalized fractional differential systems, ESAIM: Proc. 5 (1998) 145-158.

[8] M. Dunau, Représentations diffusives de seconde espèce: introduction et expérimentation, Master's Thesis, DEA d'Automatique, Toulouse, 2000.

[9] H. Zwart, Transfer functions for infinite-dimensional systems, Systems Control Lett. 52 (3-4) (2004) 247-255.

[10] H. Haddar, T. Hélie, D. Matignon, A Webster-Lokshin model for waves with viscothermal losses and impedance boundary conditions: strong solutions, in: Proceedings of the 6th International Conference on Mathematical and Numerical Aspects of Wave Propagation Phenomena, INRIA, Jyväskylä, Finland, 2003, pp. 66-71.

[11] T. Hélie, D. Matignon, Numerical simulation of acoustic waveguides for Webster-Lokshin model using diffusive representations, in: Proceedings of the 6th International Conference on Mathematical and Numerical Aspects of Wave Propagation Phenomena, INRIA, Jyväskylä, Finland, 2003, pp. 72-77.
[12] T. Hélie, D. Matignon, Diffusive representations for the analysis and simulation of flared acoustic pipes with viscothermal losses, Math. Models Meth. Appl. Sci. 16 (4) (2006) $1-34$.

[13] D. Heleschewitz, Analyse et simulation de systèmes différentiels fractionnaires et pseudo-différentiels linéaires sous représentation diffusive, Ph.D. Thesis, ENST, Paris, December 2000.

[14] O.J. Staffans, Well-posedness and stabilizability of a viscoelastic equation in energy space, Trans. Amer. Math. Soc. 345 (2) (1994) 527-575.

[15] D. Matignon, H. Zwart, Diffusive systems as well-posed linear systems, Systems Control Lett., submitted.

[16] G. Dauphin, D. Heleschewitz, D. Matignon, Extended diffusive representations and application to non-standard oscillators, in: Proceedings of the Mathematical Theory of Networks and Systems Symposium, MTNS, Perpignan, France, 2000, 10pp (invited session).

[17] G. Mittag-Leffler, Sur la représentation analytique d'une branche uniforme d'une fonction monogène, Acta Math. 29 (1904) 101-168.

[18] J. Audounet, D. Matignon, G. Montseny, Opérateurs différentiels fractionnaires, opérateurs pseudo-différentiels, représentations diffusives, Technical Report 99501, LAAS, 1999.

[19] C. Bonnet, J.R. Partington, Coprime factorizations and stability of fractional differential systems, Systems Control Lett. 41 (2000) 167-174.

[20] C. Bonnet, J.R. Partington, Analysis of fractional delay systems of retarded and neutral type, Automatica 38 (7) (2002) 1133-1138.

[21] G. Garcia, J. Bernussou, Identification of the dynamics of a lead acid battery by a diffusive model, ESAIM: Proc. 5 (1998) 87-98.

[22] E. Walter, L. Pronzato, Identification of Parametric Models from Experimental Data, Springer, Berlin, 1997. 